\title{
THE TRANSFORMATION OF BASIC SETS OF POLYNOMIALS
}

\section{N. M. MIKHAIL}

1. Introduction. The subject of basic sets of polynomials was introduced by J. M. Whittaker. For such a set $\left\{p_{n}(z)\right\}$, any polynomial and in particular, the polynomial $z^{n}$, admits a unique finite representation of the form:

$$
z^{n}=\sum_{i} w_{n i} p_{i}(z)=\sum_{i} w_{n i} \sum_{j} p_{i j} z^{j},
$$

where $\left(w_{i j}\right),\left(p_{i j}\right)$ are the basic sets of operators and coefficients respectively, associated with $\left\{p_{n}(z)\right\}$.

The basic set of polynomials is effective on $|z|=R$ if it represents every function regular in $|z|=R$.

Whittaker investigated the order and the type of the basic set $\left\{p_{n}(A z+H)\right\}(A \neq 0)$, in terms of the order and the type of the basic set $\left\{p_{n}(z)\right\}$.

Here, we investigate the effectiveness of the transformations of a given basic set of polynomials $\left\{p_{n}(z)\right\}$ i.e. the effectiveness of the sets $\left\{p_{n}(A z+H)\right\}$ for some values of $A$ and $H$, and the relation between their domains of effectiveness. We prove the following two theorems:

THEOREM 1. Let $\left\{p_{n}(z)\right\}$ be a basic set of polynomials effective on $|z|=R$, where $R \geqq b$; then the basic set $\left\{p_{n}(A z+H)\right\}$, is:

(i) effective on $|z|=R$, for all values of $R$ satisfying $b \leqq R$ if $|H| \leqq b$,

(ii) effective nowhere if $|H|>b$.

THEOREM 2. Let $\left\{p_{n}(z)\right\}$ be a basic set of polynomials effective on $|z|=R$, where $b \leqq R \leqq B$; then the basic set $\left\{p_{n}(A z+H)\right\},|H| \leqq b$, is effective on $|z|=R$ where $R$ satisfies $b / A \leqq R \leqq B / A$.

Thus, we find that, if the given set is effective, then all its transformation sets are still effective if and only if the condition on $H$, stated in Theorems 1 and 2, is satisfied. Also, we find by Theorems 1 and 2 that through the transformation, it will be possible to change the boundaries of the domain of effectiveness. Thus, it will be possible to represent any function, of given radius of regularity, by one of the transformation sets of any given basic set of polynomials, which is effective somewhere.

Received by the editors October 29, 1957 and, in revised form, December 23, 1957. 
2. Notation and previous results. The author has used his own method to determine the effectiveness of basic sets of polynomials by means of some constants, which he introduced in [1]. ${ }^{1}$ These constants are $C, b$ and $B$, which when associated with the set $\left\{p_{n}(z)\right\}$, are defined by:

(2.1) $C=\sup _{d(n)}\left(\lim \sup _{n}\left(\sup _{i}\left(\left|w_{n i}\right|\left|p_{i d(n)}\right|\right)^{1 / n}\right)\right)$, where $(d(n))$ is a sequence of integers such that: $\lim \sup _{n} d(n) / n=1$.

(2.2) $\quad b=\sup _{s(n)}\left(\lim \sup _{n}\left(\sup _{i}\left(\left|w_{n i}\right|\left|p_{i s(n)}\right|\right)^{1 / n-s(n)}\right)\right)$, where $(s(n))$ is a sequence of integers such that: $\lim \sup _{n} s(n) / n=\sigma<1$.

(2.3) $B=\inf _{g(n)}\left(\lim \sup _{n}\left(\sup _{i}\left(\left|w_{n i}\right|\left|p_{i g(n)}\right|\right)^{1 / n-g(n)}\right)\right)$, where $(g(n))$ is a sequence of integers such that: $\lim \sup _{n} g(n) / n=\sigma^{\prime}>1$.

In case of the reciprocal set $\left\{p_{n}^{*}(z)\right\}$, the associated constants are $C^{*}, b^{*}$ and $B^{*}$ and they are defined by interchanging the operators and the coefficients in (2.1), (2.2) and (2.3) respectively.

If $\left\{u_{n}(z)\right\}$ is the product set of the sets $\left\{p_{n}(z)\right\}$ and $\left\{q_{n}(z)\right\}$ in this order, i.e.

$$
\left\{u_{n}(z)\right\}=\left\{p_{n}(z)\right\}\left\{q_{n}(z)\right\},
$$

the corresponding constants $C_{u}, b_{u}$ and $B_{u}$ are defined by:

$C_{u}=C_{p q}=\sup _{d(n)}\left(\lim \sup _{n}\left(\sup _{m i x}\left(\left|v_{n m}\right|\left|w_{m i}\right|\left|p_{i x}\right|\left|q_{x d(n)}\right|\right)^{1 / n}\right)\right)$, and so on for $b_{u}$ and $B_{u}$, where $\left(v_{i j}\right)$ and $\left(q_{i j}\right)$ are the operators and the coefficients sets, respectively, associated with the set $\left\{q_{n}(z)\right\}$.

The author, has introduced in [1], the notation of quotient sets. If

$$
\left\{u_{n}(z)\right\}=\left\{p_{n}(z)\right\}\left\{q_{n}(z)\right\},
$$

then the set $\left\{p_{n}(z)\right\}$ is the quotient set of $\left\{u_{n}(z)\right\}$ and $\left\{q_{n}(z)\right\}$ in this order i.e.

$$
\left\{p_{n}(z)\right\}=\left\{u_{n}(z)\right\}\left\{q_{n}^{*}(z)\right\},
$$

and accordingly, we can find the corresponding constants $C_{p}, b_{p}$ and $B_{p}$ which are actually $C_{u q^{*}}, b_{u q^{*}}$, and $B_{u q^{*}}$, respectively.

We proved in [1], that for any basic set of polynomials, if $C \leqq 1$, then such a set is effective on $|z|=R$, where $R$ satisfies:

(i) $b \leqq R$, when lim $\sup _{n} D_{n} / n=1$,

(ii) $b \leqq R \leqq B$, when lim $\sup _{n} D_{n} / n$ is greater than unity. $\left(D_{n}\right.$ is the highest power of $z$ in (1.1).)

We use, here, the notation $s(n ; p)$ and $s(n ; u)$ to discriminate be-

\footnotetext{
1 The numbers in square brackets refer to the references at the end of the paper.
} 
tween the numbers of the sequence $(s(n))$ used in finding the values of $b$ and $b_{u}$ respectively. The same is followed in case of $(g(n))$.

\section{Proof of Theorem 1.}

(i) If $|H| \leqq b$,

write $\left\{u_{n}(z)\right\}$ for the transformation set $\left\{p_{n}(A z+H)\right\}$. Let the set $\left\{q_{n}(z)\right\}$ be a basic set of polynomials defined by:

$$
q_{n}(z)=(A z+H)^{n} \text {, for every value of } n \text {. }
$$

Let $U, P$ and $Q$ be the matrices of coefficients, $Y, W$ and $V$ the matrices of operators of the sets $\left\{u_{n}(z)\right\},\left\{p_{n}(z)\right\}$ and $\left\{q_{n}(z)\right\}$ respectively. We can easily find that $U=P Q$. So the set $\left\{u_{n}(z)\right\}$ is actually the product set of the two sets $\left\{p_{n}(z)\right\}$ and $\left\{q_{n}(z)\right\}$ in this order i.e.

$$
\left\{u_{n}(z)\right\}=\left\{p_{n}(z)\right\}\left\{q_{n}(z)\right\} .
$$

Also, we have:

$$
\left\{p_{n}(z)\right\}=\left\{u_{n}(z)\right\}\left\{q_{n}^{*}(z)\right\} .
$$

Hence, in view of (3.1) and (2.1), we have:

$C_{u}=C_{p q}=\sup _{d(n)}\left(\lim \sup _{n}\left(\sup _{m i x}\left(\left|v_{n m}\right|\left|w_{m i}\right|\left|p_{i x}\right|\left|q_{x d(n)}\right|\right)\right)^{1 / n}\right)$.

But, we know that:

$$
v_{n m}=\left(\begin{array}{c}
n \\
m
\end{array}\right)(H / A)^{n} H^{-m}, \text { and } q_{x j}=\left(\begin{array}{c}
x \\
j
\end{array}\right) A^{j} H^{x-j} .
$$

Hence, $C_{u} \geqq \lim \sup _{n}\left(\left|v_{n n}\right|\left|w_{n i}\right|\left|p_{i d(n)}\right|\left|q_{d(n) d(n)}\right|\right)^{1 / n}$, i.e.

$$
C_{u} \geqq C \text {. }
$$

Also, in view of (3.2) and (2.1), we have:

$$
\begin{gathered}
C \geqq \lim \sup _{n}\left(\left|q_{n n}\right|\left|y_{n i}\right|\left|u_{i d(n)}\right|\left|v_{d(n) d(n)}\right|\right)^{1 / n}, \text { i.e. } \\
C \geqq C_{u} .
\end{gathered}
$$

Combining the results (3.3) and (3.4), we get: $C_{u}=C$. Since $C \leqq 1$ because of the effectiveness of the set $\left\{p_{n}(z)\right\}$, then we have: $C_{u} \leqq 1$. Hence the transformed set $\left\{u_{n}(z)\right\}$ satisfies the necessary condition of effectiveness [1, Theorem $5(\mathrm{a})]$.

In view of (3.1) and (2.2), we have:

$$
b_{u}=\sup _{8(n)}\left(\lim \sup _{n}\left(\sup _{m i x}\left(\left|v_{n m}\right|\left|w_{m i}\right|\left|p_{i x}\right|\left|q_{x s(n)}\right|\right)^{1 / n-8(n)}\right) .\right.
$$

This equality holds for every possible value of $m, i$ and $x$ because $|H| \leqq b$. Hence: 


$$
b_{u} \geqq b\left(A^{-n} A^{s(n)}\right)^{1 / n-s(n)}=b / A .
$$

Also, in view of (3.2) and (2.2), we have by similar treatment:

$$
\begin{aligned}
b & \geqq b_{u} A, \text { i.e. } \\
b_{u} & \leqq b / A .
\end{aligned}
$$

Combining the results (3.5) and (3.6), we get the following result;

$$
b_{u}=b / A \text {. }
$$

Thus the transformed set $\left\{p_{n}(A z+H)\right\}$ is effective on $|z|=R$, where $R$ is such that $R \geqq b / A$.

For example, if the basic set of polynomials $\left\{p_{n}(z)\right\}$ is effective in all the discs $R \geqq b$, then the translated set $\left\{p_{n}(z+H)\right\}$ is still effective for the same discs if $|H| \leqq b$.

(ii) If $|H|>b$, for simplicity, put $A=1$. Then we have in view of (3.2) and (2.2):

$$
\begin{aligned}
& b \geqq \lim \sup _{n}\left(\left|q_{n n}\right|\left|y_{n i}\right|\left|u_{i s(n ; u)}\right|\left|v_{s(n ; u) 0}\right|\right)^{1 / n} \\
& \geqq \lim \sup _{n}\left(b_{u}\right)^{(n-8(n ; u)) / n}|H| s(n ; u) / n \text {, i.e. } \\
& b>b_{u} \text {, since }|H|>b \text {. }
\end{aligned}
$$

Also, in view of (3.1) and (2.2), we have:

$$
\begin{gathered}
b_{u} \geqq \lim \sup _{n}(b)^{(n-s(n ; p)) / n}|H|^{s(n ; p) / n}, \text { i.e. } \\
b_{u}>b .
\end{gathered}
$$

As it is impossible to find such a value for $b_{u}$ satisfying both inequalities (3.7) and (3.8), then the set $\left\{u_{n}(z)\right\}=\left\{p_{n}(A z+H)\right\}$ is effective nowhere, for every value of $|H|>b$. E.g. consider the simple set $\left\{p_{n}(z)\right\}$ defined by: $p_{n}(z)=z^{n}$, for every value of $n$. We know that this set is effective in the whole plane, i.e. $b=0$. Thus, we expect that for any value of $H$, the translated set $\left\{p_{n}(z+H)\right\}$ is effective nowhere, and this is true.

Proof of Theorem 2. From Theorem 1, we have:

$$
C_{u}=C \text { and } b_{u}=b / A \text {. }
$$

Regarding the value of $B_{u}$, we have in view of (3.1) and (2.3):

$$
\begin{gathered}
B_{u} \leqq \lim \sup _{n}\left(\left|v_{n n}\right|\left|w_{n i}\right|\left|p_{i g(n ; p)}\right|\left|q_{o(n ; p) \vartheta(n ; p)}\right|\right)^{1 / n-o(n ; p)}, \text { i.e. } \\
B_{u} \leqq B\left(A^{-n+o(n ; p)}\right)^{1 / n-o(n ; p)}=B / A .
\end{gathered}
$$

Also, in view of (3.2) and (2.3), we have:

$$
\begin{aligned}
B & \leqq B_{u}\left(A^{n-g(n ; u)}\right)^{1 / n-g(n ; u)}=B_{u} A, \text { i.e. } \\
B_{u} & \geqq B / A .
\end{aligned}
$$


Combining the results (3.9) and (3.10), we get:

$$
B_{u}=B / A \text {. }
$$

Thus the result of the theorem follows. N.B. The results $C_{u}=C$ and $b_{u}=b / A$, proved in Theorem 1 , and employed in the proof of the above theorem hold for every value of $m, i$ and $x$ because of the imposed condition $|H| \leqq b$. For example when investigating the value of $b_{u}$, we find that $x$ may belong to the sequence $(d(n))$ or the sequence $(g(n))$.

If $x$ belongs to $(d(n))$, we have: $b_{u} \geqq|H| C^{1 /(1-\sigma)} / A$. It is obvious that the R.H.S. of this inequality is still less or equal to $b / A$. Thus the inequality $b_{u} \geqq b / A$ deduced in Theorem 1 , in which $x$ belongs to $(s(n))$, still holds in this case. If $x$ belongs to $(g(n))$, we have: $b_{u} \geqq|H|^{(\dot{\sigma}-\sigma) /(1-\sigma)} / A B^{(\dot{\sigma}-1) /(1-\sigma)}$. The R.H.S. of this inequality is still less or equal to $b / A$. Thus the inequality $b_{u} \geqq b / A$ holds always.

We, also, conclude that the condition $|H| \leqq b$ is sufficient and necessary for the effectiveness of the transformed set $\left\{p_{n}(A z+H)\right\}$ where the set $\left\{p_{n}(z)\right\}$ is effective somewhere. The sufficiency follows from Theorem 1(i) and Theorem 2, and the necessity follows from Theorem 1(ii).

\section{REFERENCES}

1. M. N. Mikhail, Basic sets of polynomials and their reciprocal, product and quotient sets, Duke Math. J. vol. 20 (1953) pp. 459-480.

2. J. M. Whittaker, Sur les series base de polynomes quelconque, Gauthier-Villars, Paris, 1949.

3. - Interpolatory function theory, Cambridge Tracts in Mathematics and Physics, no. 33, 1935.

Ein Shams University, Cairo 